\title{
New Sirolimus-Coated Ballon for Complex Unprotected Left Main Distal Bifurcation
}

\author{
Gaetano di Palma ${ }^{1}$ and Bernardo Cortese ${ }^{2 *}$ \\ Unità Operativa di Cardiologia, Italy
}

Received: February 02, 2018; Published: February 15, 2018

*Corresponding author: Bernardo Cortese, Interventional Cardiology, ASST Fatebenefratelli-Sacco, Milano, Italy, Email: bcortese@gmail.com

\begin{abstract}
Treatment of unprotected left main (ULM) in-stent restenosis (ISR) is still highly challenging for interventional cardiologists; data are limited and the optimal strategy still debated. Drug-coated balloons (DCB) have an acknowledged role for ISR treatment. Until 2016 all DCB available in Europe eluted paclitaxel; since then, a new device coated with sirolimus, the Magic Touch区 (Envision Scientific PVT, India), has been marketed in Europe. This device also shares new technological characteristics that render it appealing for complex cases. We here present the first case of distal bifurcation ULM ISR treated with sirolimus-coated balloons simultaneous insufflation with a kissing-balloon technique.
\end{abstract}

Keywords: Sirolimus-coated balloon; In-stent restenosis; Unprotected left main

\section{Introduction}

Treatment of unprotected left main (ULM) in-stent restenosis (ISR) is a tricky intervention, often burdened by high recurrencies. Until now, limited data exist on the optimal treatment strategy. Drug-coated balloons (DCB) represent a well established treatment for both bare-metal stent (BMS) and drug-eluting stent (DES) restenosis [1]. Until 2016, all DCBs available in Europe eluted paclitaxel, an highly lipophilic drug with narrow therapeutic window. In April 2016 the first sirolimus-coated balloon (SCB), Magic Touch $\square$ (Envision Scientific PVT, India), obtained the CE Mark. This SCB is the first to elute sirolimus, thanks to a phospolipid nanocarrier system that allows long term drug retention in the vessel wall [2]. We here present the first case in Europe of ULM bifurcation ISR treated with a kissing-balloon technique using two SCBs.

\section{Case Report}

A 48-year-old male arrived to our hospital with a non STelevation myocardial infarction. He had a well known history of coronary artery disease, having already received several percutaneous coronary interventions (PCI). Three years before the patient received a $3.5 / 12$ DES in the left main-left anterior descending artery (LAD) because of an acute coronary syndrome. Six months later, he experienced recurrent angina, and coronary angiography showed ISR of the distal LM-ostial LAD, that was treated with a 3.0/18 DES. After 8 months the patient presented with UA, and coronarography showed a sub-occlusive ISR of the distal LM-ostial LAD, with significant stenosis of the ostial left circumflex artery (LCA); the patient refused coronary artery bypass and the lesion was treated with a 3.5/30 paclitaxel-coated balloon of LM-LAD, with plaque shift on the proximal LCA, where a new 3/8 DES was implanted. A scheduled coronary angiography 6 months later showed a good result of the previous PCI (Figure 1).

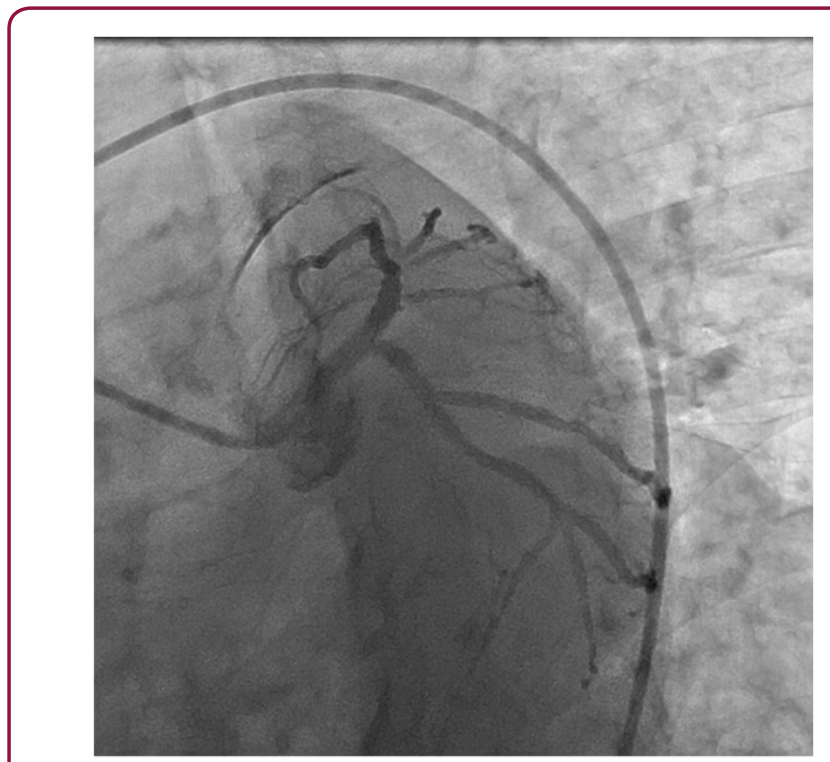

Figure 1: Persisting good result of left main PCI with drug-coated balloon after 6 months. 
During index procedure, that occurred 8 months later, coronary angiography showed $80 \%$ ISR of distal LM involving the ostial LAD and LCA (Video 1). Optical-coherence tomography (OCT) (Video 2) analysis of the LM-LAD showed under-expansion of the EES previously implanted on the LM with significant neointimal hyperplasia; minimal lumen area (MLA) of ostial LAD was $1.41 \mathrm{~mm} 2$ and the ostium of LCA was not visible (Figure 2). The procedure was performed through a right radial acces with a $6 \mathrm{~F}$ guiding catheter. Both the LAD and LCA were wired and a kissing-balloon pre-dilation was performed using non-compliant balloons, 3.5/15 for the LM-LAD and $2.75 / 15 \mathrm{~mm}$ for the LCA. The angiography showed an adequate preparation of both lesions; therefore also in consideration of the several stent struts apposed, we programmed a only-DCB PCI. Two SCBs were simultaneously inflated with kissing-balloon technique (3.5/15 in the LM-LAD and $2.5 / 20 \mathrm{~mm}$ in the LCA) (Figure 3). Final angiography showed absence of residual stenosis with TIMI 3 flow (Video 3). Final OCT analysis (Video 4) confirmed the good result of the angioplasty with good expansion of the previously implanted DES on the LM; final MLA of the distal LM was $8.9 \mathrm{~mm} 2$ and MLA of the proximal LAD was $5.8 \mathrm{~mm} 2$ (Figure 4). The patient was discharged after two days asymptomatic on dual anti-platelet therapy.

Movie 1: coronary angiography showing ISR of distal LM-ostial LAD and ostial LCA during index procedure.

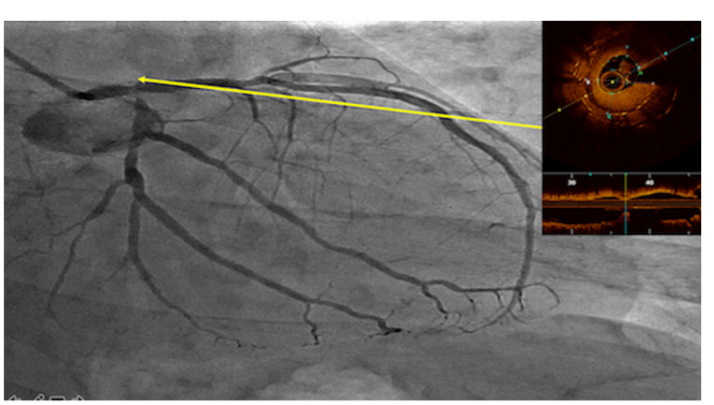

Figure 2: ISR of distal LM-ostial LAD and ostial LCA during index procedure. The OCT shows the double metal layer and under-expansion of the EES previously implanted, with a minimal lumen area (MLA) of ostial LAD of $1.41 \mathrm{~mm}$. Neointimal hyperplasia.
Movie 2: OCT showing the double metal layer and underexpansion of the EES previously implanted, with a minimal lumen area (MLA) of ostial LAD of $1.41 \mathrm{~mm}$. Neointimal hyperplasia.

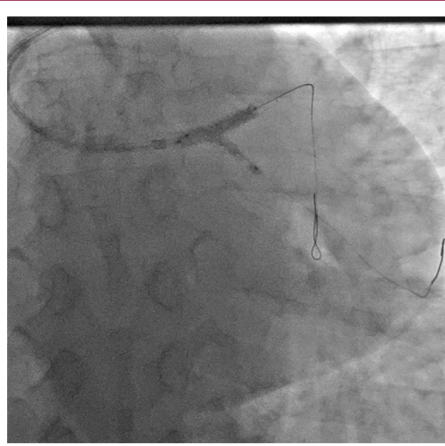

Figure 3: Kissing-balloon with two Magic Touch SCB, 3.5/15 $\mathrm{mm}$ in LM-LAD and 2.5/20 $\mathrm{mm}$ in LCA.

Movie 3: OCT after PCl showing good expansion of the DES previously implanted and a MLA of distal LM $8.9 \mathrm{~mm} 2$ and 5.8 $\mathrm{mm} 2$ for LAD. 


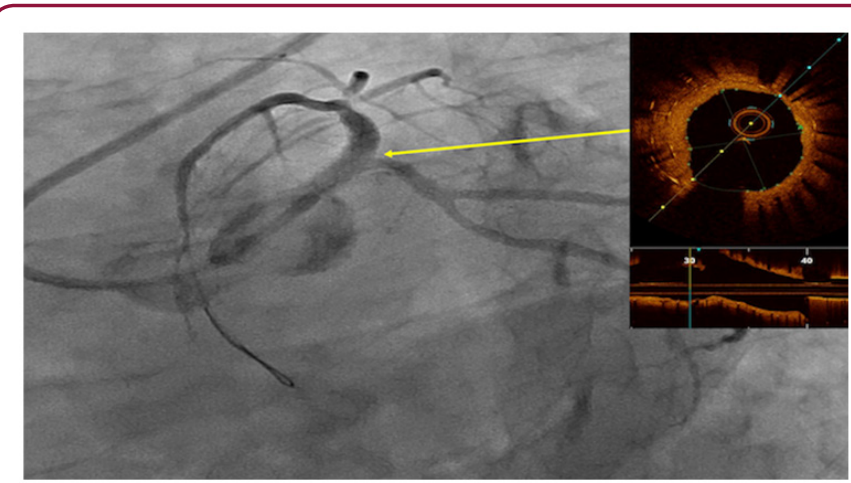

Figure 4: Final angiographic result. The OCT analysis shows a good expansion of the DES previously implanted and a MLA of distal LM $8.9 \mathrm{~mm} 2$ and $5.8 \mathrm{~mm} 2$ for LAD.
Movie 4: final angiographic result with TIMI flow 3, after PCI.

\section{Discussion}

DCB are widely used for ISR management1 and are also variably used in other settings (small vessels, bifurcation) (3-5). Limited data are currently available on the optimal management of LM ISR, and the few available seem to suggest a good outcome of second generation DES $(6,7)$. DCB with kissing balloon technique for LM bifurcation is an attractive concept, avoiding additional metal layers in a delicate area, where an excessive shear stress may be responsible for late thrombotic events. Moreover, adding further metal struts may also modify the bifurcation carina. Compared to paclitaxel, sirolimus is less lipophilic, has a wider therapeutic window (8) and the unique nano-encapsulation process of the SCB with the phospholipid drug carrier allows an improved drug retention profile and bioavailability in atherosclerotic or restenotic lesions (2). Furthermore the latest-generation monorail delivery system, compatible with 5-Fr guiding catheters, the low-profile distal tip and the rigid hypotube, warrant an high deliverability and trackability, and make it possible to use 2 balloons contemporarily during kissing balloon inflation, reducing the risk of struts distortion with a normal 2-step inflation, as previously demonstrated by Shetty et al. [9].

In our patient the presence of 2 metal layers on LM-LAD, along with the failure of last generation EES was the main reason for a DCB strategy. The previous failure of PCBs on both LCA and LM$\mathrm{LAD}$, and the possibility to perform a kissing balloon with the Magic Touch device, were the reasons we decided for a SCB. To this day, limited data are available on the long term efficacy of SCB [10].

\section{Conclusion}

This is the first case of a LM ISR bifurcation treated with a SCB PCI with kissing ballon technique in Europe. Considering the recognized drug efficacy and safety profile, and the high deliverability and trackability of the device, the use of SCB could represent an attractive approach for complex ISRs.

\section{References}

1. Windecker S, Kolh P, Alfonso F, Collet J-P, Cremer J, et al. (2015) 2014 ESC/ EACTS guidelines on myocardial revascularization. EuroIntervention 10(9):1024-1094.

2. Sojitra P, Yazdani S, Otsuka F, Doshi M, Kolodgie F, et al. (2013) CRT123 A Novel Nano Particle Sirolimus Delivery Via Coated Balloon. JACC Cardiovasc Interv 6: S40.

3. Cortese B, Bertoletti A (2012) Paclitaxel coated balloons for coronary artery interventions: a comprehensive review of preclinical and clinical data. Int J Cardiol 161(1): 4-12.

4. Loh JP, Barbash IM, Waksman R (2013) The current status of drugcoated balloons in percutaneous coronary and peripheral interventions. EuroIntervention 9(8): 979-988.

5. Cortese B, Berti S, Biondi-Zoccai G, Colombo A, Limbruno U, et al. (2014) Drug-coated balloon treatment of coronary artery disease: a position paper of the Italian Society of Interventional Cardiology. Catheter Cardiovasc Interv 83(3): 427-435.

6. De Caterina AR, Cuculi F, Banning AP (2013) Incidence, predictors and management of left main coronary artery stent restenosis: a comprehensive review in the era of drug-eluting stents. EuroIntervention 8(11): 1326-1334.

7. D’Ascenzo F, Chieffo A, Cerrato E, Ugo F, Pavani M, et al. (2017) Incidence and Management of Restenosis After Treatment of Unprotected Left Main Disease With Second-Generation Drug-Eluting Stents (from Failure in Left Main Study With $2^{\text {nd }}$ Generation Stents-Cardiogroup III Study). Am J Cardiol 119(7): 978-982.

8. Lemos PA, Farooq V, Takimura CK, Gutierrez PS, Virmani R, et al. (2013) Emerging technologies: polymer-free phospholipid encapsulated sirolimus nanocarriers for the controlled release of drug from a stentplus-balloon or a stand-alone balloon catheter. EuroIntervention 9(1): 148-156.

9. Shetty R, Ganiga Sanjeeva NC, Agarwal S, Doshi M, Sojitra P (2015) Unprotected distal left main bifurcation drug eluting stent restenosis: first successful experience with simultaneous kissing balloon dilatation using sirolimus coated balloon. Cardiovasc Diagn Ther 5(6): 484-487.

10. Cortese B, di Palma G, Latini RA, Elwany M, Orrego PS (2017) Immediate and short term performance of a novel sirolimus-coated balloon during complex percutaneous coronary interventions. The FAtebenefratelli SIrolimus COated-balloon (FASICO) registry. Cardiovasc Revasc Med 18(7): 487-491. 
(c) (i) This work is licensed under Creative Submission Link: http://biomedres.us/submit-manuscript.php

\begin{tabular}{ll} 
BIOMEDICAL & Assets of Publishing with us \\
RESEARCHES & - Global archiving of articles \\
\hline ISSN:2574-1241 & - Immediate, unrestricted online access \\
\hline
\end{tabular}

